\title{
Quiste broncogénico. Reporte de un caso y revisión de la literatura
}

\author{
Miguel Cosío-Pascal, * ${ }^{*}$ Ludmila Cosío-Lima, ${ }^{*}$ Carlos Alberto Lezama-Urtecho, ${ }^{\S}$ José Luis Ríos-Reyna"
}

*Neumología-Cardiología, Hospital Ángeles Mocel; ${ }^{\ddagger}$ Associate Professor Exercise Sciences. Department of Health, Leisure and Exercise Sciences. University of West Florida, FL, USA; ${ }^{\circledR}$ Cirugía cardiotorácica, Hospital Ángeles Mocel; \#Jefe de Imagenología y Hemodinámica, Hospital Ángeles Mocel

Trabajo recibido: 22-XI-2013; aceptado: 04-II-2014

RESUMEN. El objetivo de la presente comunicación es presentar el caso de un paciente de 78 años con quiste broncogénico (QB) que ocupaba dos espacios mediastinales; no obstante el tamaño de la anomalía, no había presentado sintomatología. Inició el cuadro clínico con dolor retroesternal discreto, pero persistente. Se le realizó radiografía de tórax en la cual se interpretó erróneamente la presencia de «cardiomegalia». La tomografía computarizada mostró que el QB oprimía el corazón hacia adelante, lo que seguramente originó el síntoma inicial. Los QB son malformaciones congénitas poco frecuentes. El sistema respiratorio se origina a partir de un brote ventral del intestino primitivo durante la séptima semana de gestación. Los QB se organizan a partir de células que se separan del brote respiratorio inicial conservando las características del epitelio respiratorio. La mayoría de los QB se localizan en el tórax, ya sea en el mediastino o en el parénquima pulmonar, existiendo con mucha menor frecuencia casos en localizaciones atípicas.

Otro de los objetivos es llamar la atención acerca de esta patología tan poco frecuente, su embriogénesis y los cuadros clínicos extraordinariamente variados que se originan de acuerdo con su tamaño y localización, de suerte tal que puede involucrar a médicos de muy distintas especialidades. La incidencia de los QB se desconoce, pueden ser asintomáticos durante toda la vida y ser eventualmente hallazgos de autopsia. Otros más permanecen asintomáticos durante períodos variables hasta iniciar el cuadro clínico, o bien, pueden ser descubiertos en forma incidental por estudios de imagenología. Se hizo una revisión de la literatura, principalmente la publicada por instituciones reconocidas a nivel mundial, habiendo encontrado que el caso registrado con mayor edad al iniciar la sintomatología fue de 72 años. El paciente que presentamos en esta comunicación tenía 78 años al empezar a presentar dolor retroesternal, situación que lo constituye como el caso reportado en la literatura mundial que ha permanecido durante más años asintomático. Fue operado; habiendo extraído el tumor, se encuentra bien y asintomático.

Palabras clave: Quiste broncogénico, embriología, cuadros clínicos, casos atípicos.

ABSTRACT. We are presenting a case of a 78-year-old patient with a bronchogenic cyst (BC) which was located between two mediastinum spaces and even though the anomaly, the patient was asymptomatic. The patient presented with persistent retrosternal pain and the thoracic X-ray wrongly interpreted the presence of «cardiomegaly». The computed tomography showed that the BC was pressing the heart forward, which could have been the cause of the original symptoms.

Bronchogenic cysts are rare and infrequent congenital aberrations. The respiratory system develops from a ventral diverticulum of the foregut during the seventh week of gestation. The pharynx and esophagus are originated from the caudal region of this structure. The BC will develop from cells that separate from the initial tracheobronchial tree conserving the characteristics of the bronchial respiratory epithelium. Most of the $\mathrm{BC}$ are located in the thorax, either in the mediastinum or in the pulmonary parenchyma, but there are also very rare reports of BC in atypical locations. A secondary purpose of this review is to discuss the pathology, embryology and clinical pictures of these rare occurrences. According to their size and location, they will require the expertise of physicians of different specialties. The exact incidence of the BC is not known, since there are asymptomatic cases that eventually are found through autopsies or in patients that were asymptomatic during variable periods of time or had been detected through an incidental thoracic radiography or other imageology studies. In the reviewed literature the oldest case reported was a male that presented symptoms at 72 years of age. In this article the patient is a 78 -year-old male that presented symptoms at that age. We can conclude that this patient is the case reported in the literature that remained asymptomatic for the longest period of time. The patient had surgery and the $\mathrm{BC}$ was extracted; the patient is in stable condition and asymptomatic.

Key words: Bronchogenic cysts, embryology, diagnosis, atypical cases.

\section{INTRODUCCIÓN}

Los quistes broncogénicos (QB) son formaciones congénitas que derivan del desarrollo fetal anómalo del árbol traqueobronquial. Los órganos del sistema respiratorio y el esófago tienen como origen común el intestino primitivo. Durante la séptima semana de gestación este conducto se alarga verticalmente, apareciendo un brote ventral a partir del cual se desarrollará el sistema respiratorio. La porción caudal dará origen al tracto gastrointes- 
tinal superior. La separación de estas estructuras puede dar lugar a formaciones quísticas. Los QB se organizan a partir de células que se separan del botón respiratorio ventral conservando las estructuras características del futuro árbol bronquial. En la pared del QB se pueden identificar: tejido conectivo fibroso, cartílago, músculo liso, vasos, tejido elástico, nervios y glándulas mucosas. El epitelio del QB es de tipo secretor respiratorio, seudoestratificado, columnar y ciliado. ${ }^{1}$

La localización definitiva de estas malformaciones depende del estadio embriológico en el que se originan. Las anomalías precoces aparecen relacionadas con la tráquea, carina o bronquios principales, dando lugar a los QB de mediastino, que generalmente aparecen como estructuras únicas y de tamaño muy variado, ya que pueden medir menos de $1 \mathrm{~cm}$, llegando en ocasiones a ocupar más de un compartimento mediastinal. El contenido de estas malformaciones puede ser aire, líquido claro, material mucoso café-amarillento o sanguinolento. La presencia de oxalato de calcio da lugar a una apariencia blanquecina-lechosa y espesa. Frecuentemente se adhieren al árbol traqueobronquial mediante un pedículo, que en muy raras ocasiones comunica con las vías respiratorias. Esta formación puede presentarse también, aunque con menor frecuencia, relacionada con el esófago y la pleura. Los QB constituyen aproximadamente entre el 6 y $15 \%$ de todos los tumores del mediastino en niños y adultos, predominando en el mediastino medio. Las anomalías tardías aparecen en relación con bronquios periféricos, de tal forma que se localizan en el parénquima pulmonar. Pueden ser únicos, loculados o múltiples; con frecuencia se encuentran comunicados con las vías aéreas, razón por la cual son capaces de mimetizar a múltiples padecimientos respiratorios. Se reportan QB intraparenquimatosos con mecanismo de válvula, lo que determina que el órgano se encuentre insuflado a presión, comprometiendo a estructuras vecinas y al mismo pulmón, originando atelectasia o mimetizando neumotórax o hidroneumotórax. ${ }^{2}$

Los QB intrapulmonares se localizan con mayor frecuencia en las regiones posteriores de los lóbulos pulmonares basales. ${ }^{3,4}$ Liu et al. ${ }^{5}$ publicaron el caso de una niña de 13 años con QB parenquimatosos gigantes bilaterales en lóbulo superior derecho y lóbulo inferior izquierdo.

Otras anomalías pulmonares congénitas en relación con el intestino primitivo son los quistes adenomatoides, el enfisema lobar congénito y el secuestro pulmonar. Estas patologías en muy raras ocasiones coexisten con los QB. Grewal et al. ${ }^{6}$ reportaron el caso de un paciente con QB y secuestro pulmonar. McAdams et al. ${ }^{7}$ describieron tres casos con las mismas patologías anteriores, y Mee-
Hye et al. ${ }^{8}$ publicaron un caso con QB intrapulmonar junto con una formación adenomatoide. McKenzie et al. ${ }^{9}$ comunicaron un caso descubierto por ultrasonido (US) a las 23 semanas de gestación que comprimía el pulmón izquierdo. Se practicó aspiración percutánea del contenido quístico, que no se reprodujo durante el resto del embarazo. El neonato se operó, habiendo encontrado un secuestro pulmonar no aireado con irrigación sistémica, el QB como lesión predominante y una malformación quística adenomatoide demostrada en el examen histológico.

\section{PRESENTACIÓN DEL CASO}

Paciente de sexo masculino de 78 años de edad. Constitución fuerte, $1.72 \mathrm{~m}$ de estatura y $81 \mathrm{~kg}$ de peso. Antecedentes sin importancia. Muy deportista, desde joven practica natación y otros ejercicios aeróbicos sin problemas. Acudió al médico de primer contacto por dolor retroesternal no intenso, aunque persistente. La radiografía de tórax mostró lo que fue interpretado como «cardiomegalia», razón por la que el paciente fue referido a nuestro hospital. En la exploración física no se encontraron anomalías. El paciente consignó que nunca se le había practicado radiografía de tórax. En la placa enviada se identificaron dos imágenes superpuestas, difíciles de individualizar, que correspondían a la silueta cardíaca, que era normal. Atrás del corazón se encontraba otra imagen redondeada y tenue, cuyo perfil derecho rebasaba al de este órgano, originando el error de interpretación de «cardiomegalia». El perfil izquierdo de la imagen retrocardíaca se podía identificar difícilmente por la superposición del corazón (figura 1). La tomografía computarizada (TC) mostró la silueta quística, ovoide, con diámetro mayor de $12 \mathrm{~cm}$ con atenuación de $>20 \mathrm{HU}$, ocupando mediastino posterior y parte del medio, oprimiendo y desplazando a estructuras vecinas (figuras 2 y 3 ).

La cirugía se llevó a cabo realizando toracotomía lateral derecha. La tumoración se encontraba firmemente adherida a los lóbulos pulmonares medio e inferior mediante adherencias muy vascularizadas, así como al bronquio principal derecho y al esófago. La porción del QB adherida a esófago no se pudo resecar por completo, tratando de preservar la integridad del órgano. La pequeña porción remanente de mucosa se electrocoaguló. El contenido quístico era espeso y de color blanquecino. No era fétido, pero el cultivo demostró la presencia de estreptococo áureo, por lo que ameritó manejo antimicrobiano enérgico, manteniendo las sondas de drenaje durante 5 días. El paciente se encuentra bien y haciendo vida normal. El examen histológico confirmó el diagnóstico de QB con epitelio de tipo respiratorio en el interior del quiste (figuras 4-6). 


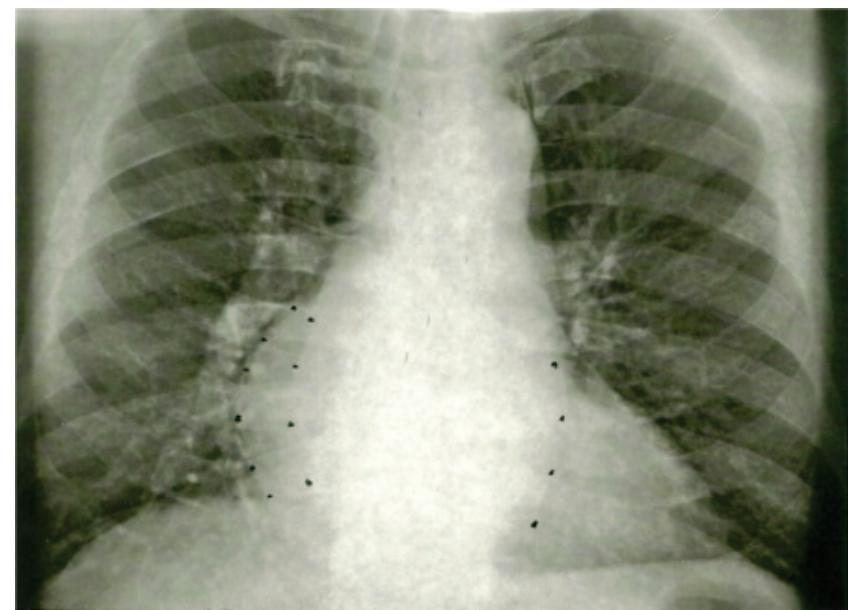

Figura 1. Radiografía PA de tórax en la que se había hecho un diagnóstico de «cardiomegalia». En realidad, se trata de dos imágenes superpuestas difíciles de identificar. La anterior es el corazón, que enmascara la imagen posterior ovoidevertical que es el QB, cuyo borde derecho simula ser el de la silueta cardíaca. Las líneas punteadas señalan la separación entre los bordes, el de la silueta cardíaca en la porción interna y la quística por fuera. El borde izquierdo del QB se identifica con los puntos correspondientes.

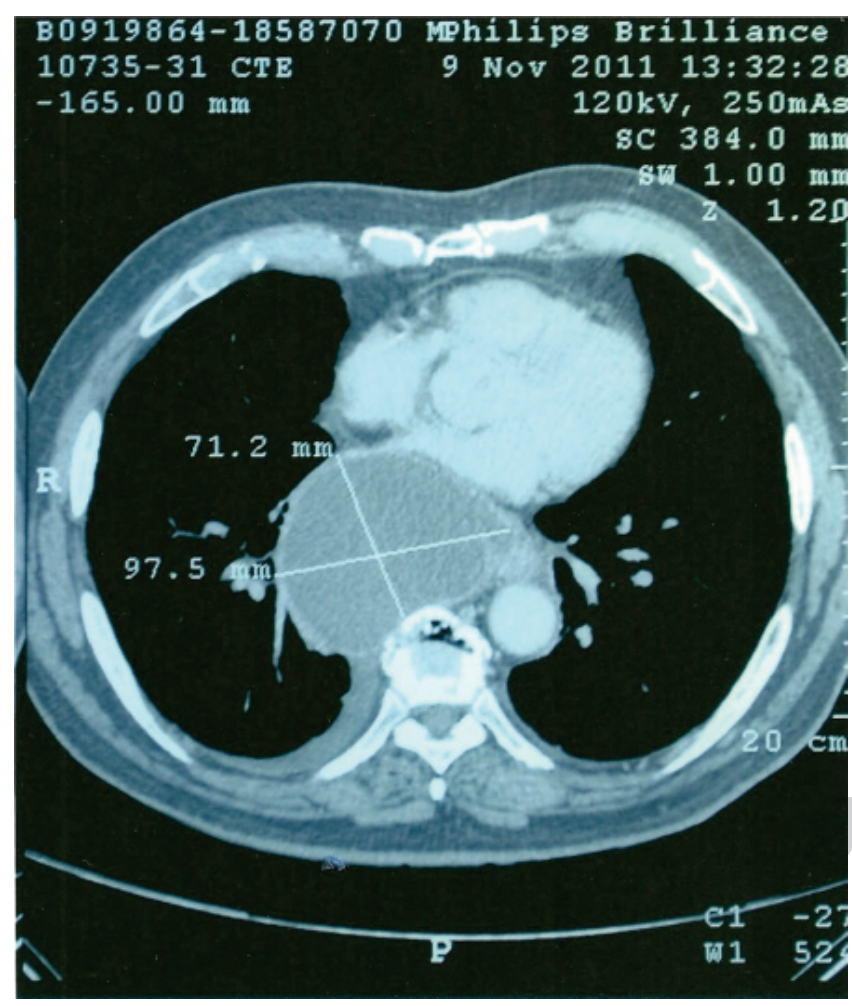

Figura 2. TAC que muestra el $\mathrm{QB}$ ocupando el mediastino posterior y parte del medio, haciendo protrusión hacia el hemitórax derecho y oprimiendo el corazón.

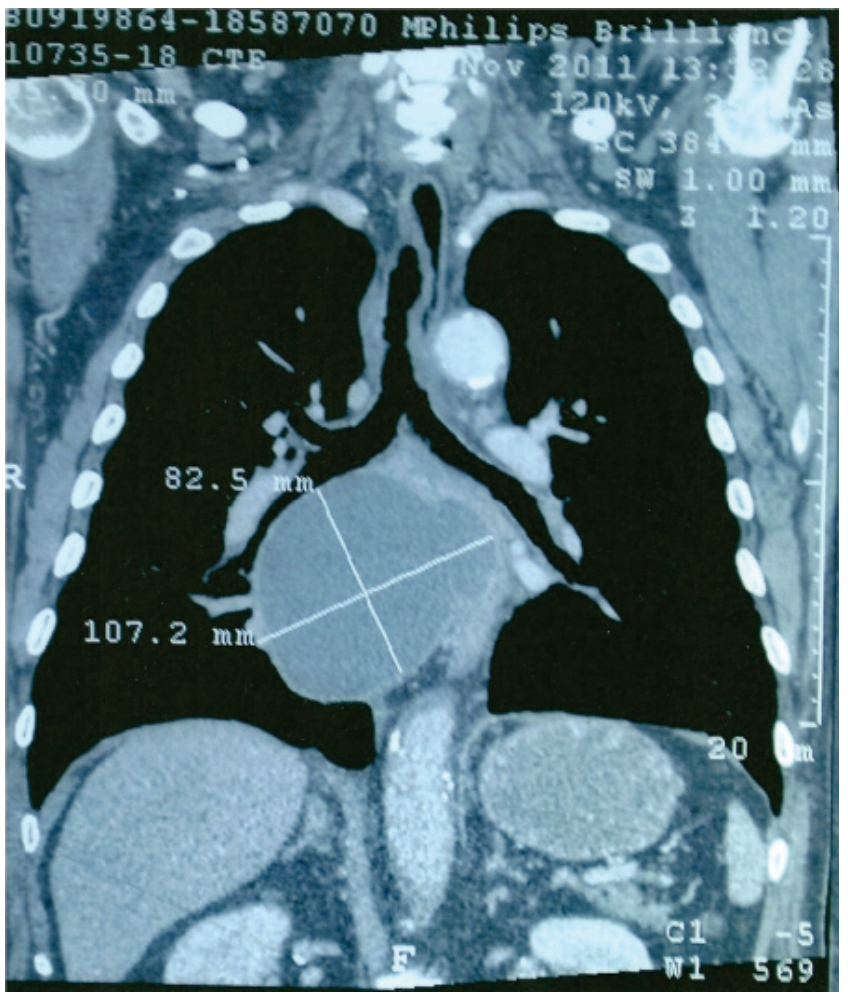

Figura 3. TC que muestra el tamaño de la malformación en íntima relación con la carina y bronquios principales, especialmente con el derecho, al que rechaza ligeramente hacia arriba y parece obstruirlo parcialmente.

\section{DISCUSIÓN}

Cuadros clínicos. En general, se puede afirmar que la sintomatología que origina el QB depende fundamentalmente de su localización y tamaño. Los localizados en el mediastino pueden ser asintomáticos durante toda la vida, pudiendo llegar a ser hallazgos de autopsia. Takeda et al. ${ }^{10}$ revisaron 105 casos de quistes del mediastino operados en el transcurso de 50 años, habiendo encontrado que los QB fueron los más numerosos, con 47 casos. El inicio del cuadro clínico puede manifestarse como molestias discretas, como es el caso que se presenta: dolor leve, disnea o disfagia que pueden agravarse con el crecimiento de la anomalía; o bien, la sintomatología inicial puede ser severa, como hemoptisis, sangrado abundante a cavidad pleural o pericárdica, ruptura del QB al interior de vías respiratorias, etcétera, y finalmente, la malformación es capaz de provocar súbitamente el fallecimiento del individuo. Un QB pequeño, pero relacionado con alguna estructura vital puede ser muy peligroso. Kennebeck et al. ${ }^{11}$ informaron de un caso que presentó síndrome coronario agudo, colapso hemodinámico e infarto cardíaco masi- 


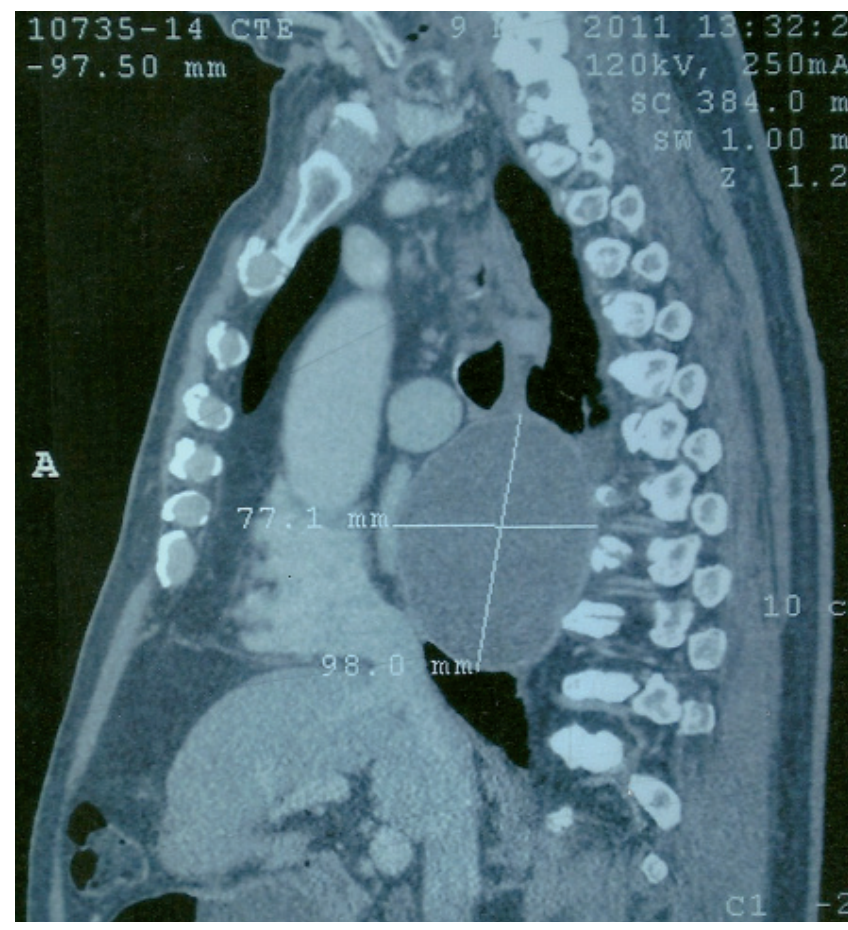

Figura 4. El QB ocupando el mediastino posterior y medio, comprimiendo hacia adelante al corazón.

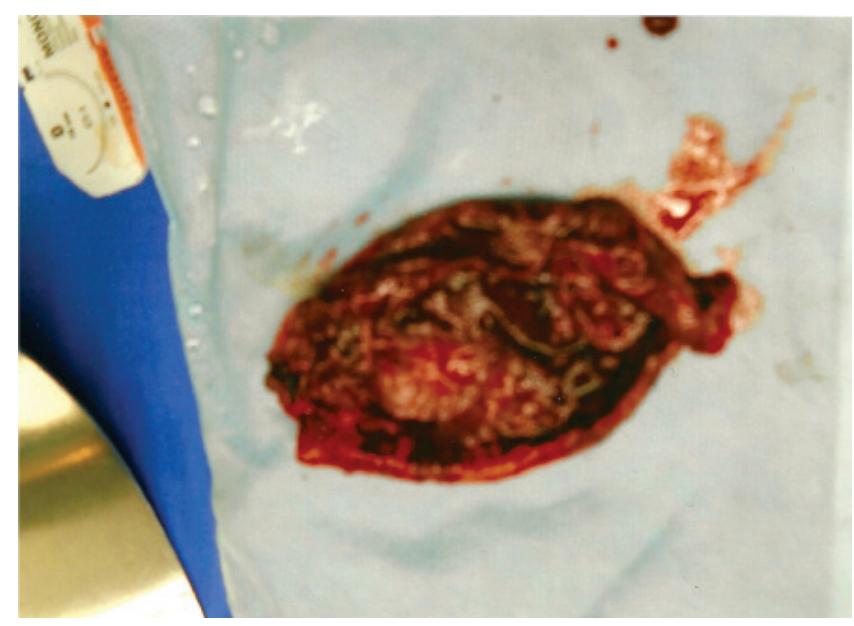

Figura 5. La pieza resecada, mostrando en su interior el material blanquecino y grumoso (oxalato de calcio).

vo. El paciente falleció, y en la autopsia se encontró un QB de menos de $1 \mathrm{~cm}$ oprimiendo y obstruyendo por completo la arteria coronaria izquierda en su origen.

Como ya se mencionó, en ocasiones los QB mediastínicos pueden relacionarse con la pleura o el esófago. ${ }^{1}$ Eom et al. ${ }^{12}$ publicaron un caso muy interesante: defecto del pericardio, secuestro pulmonar con un QB conectado al esófago por medio de una estructura tubular

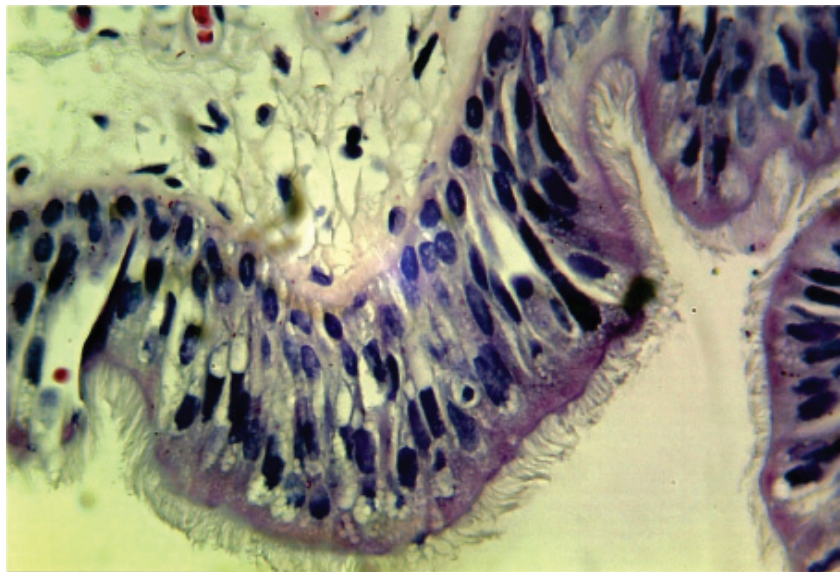

Figura 6. El epitelio que recubría el interior del quiste era típicamente respiratorio (tinción con eosina y hematoxilina, amplificación a 40x).

de $3 \mathrm{~cm}$ de longitud y $2 \mathrm{~cm}$ de diámetro. Se reportan casos operados en los que la pared de la tráquea y el QB estaban tan fusionados que parecía que ambos eran parte de la pared de la vía aérea. ${ }^{13}$

En cuanto a los QB intraparenquimatosos, Shanti et al. ${ }^{2}$ revisaron 236 quistes pulmonares resecados y encontraron que los más frecuentes fueron los $\mathrm{QB}$, con 47 casos. Se reportan QB en el parénquima pulmonar con mecanismo de válvula, lo que determina que el órgano se encuentre insuflado a presión, ${ }^{3}$ comprometiendo a las estructuras vecinas y al mismo pulmón, originando atelectasia o mimetizando neumotórax o hidroneumotórax. ${ }^{4}$ Esta situación puede constituir una seria amenaza para la vida. Hallamos en la literatura cinco casos de individuos con QB parenquimatosos con mecanismo de válvula que sufrieron barotrauma con fatales consecuencias al viajar en avión. La baja de presión durante el despegue resulta en una brusca expansión del pulmón afectado (Ley de Boyle), que literalmente estalla, permitiendo el paso del aire a los vasos pulmonares, lo que ocasionó las embolias aéreas cerebrales que terminaron con la vida de los pasajeros. ${ }^{14-18}$

Entre el $50-80 \%$ de los diagnósticos de QB se hacen en pacientes sintomáticos, especialmente en niños con localización intraparenquimatosa. En los adultos que habían permanecido asintomáticos, el cuadro clínico se presenta generalmente entre los $15-40$ años, con 38 como promedio. Los casos que inician sintomatología después de los 50 años son excepcionales.

Diagnóstico. Los estudios de imagenología son los más importantes, aunque no definitivos, para la orientación diagnóstica presuntiva, así como para definir localización, tamaño, relación con estructuras vecinas y, eventualmente, descubrir los casos asintomáticos que 
se detectan en estudios incidentales. Patel et al., ${ }^{19}$ en 18 pacientes con QB de mediastino, encontraron que en 17 de ellos, la radiografía de tórax no orientaba hacia el diagnóstico. En ocho casos se practicó TC, habiendo podido descartar únicamente en cinco la presencia de malignidad. Los autores consideraron que con el soporte clínico y los estudios de imagenología, la sospecha de QB se pudo plantear únicamente en 11 de los 18 casos, ya que el diagnóstico de certeza lo proporcionó únicamente el examen histológico de la pieza resecada. Los sujetos asintomáticos con QB en mediastino son los que se descubren incidentalmente con mayor frecuencia por medio de una radiografía de tórax, siempre y cuando la lesión no sea muy pequeña. En estos casos, la imagen es generalmente redondeada, bien delimitada, poco densa y relacionada frecuentemente con la carina. La TC es un elemento importante en relación con los QB. La pared de la anomalía congénita se observa bien delimitada, y la composición del material que contiene explica los diferentes grados de atenuación que pueden aparecer en este estudio. Aproximadamente en el $50 \%$ de los casos, la densidad del fluido que contiene varía entre $0-20 \mathrm{HU}$, pero se puede presentar atenuación característica de tejidos blandos $>30 \mathrm{HU}$, identificar oxalato de calcio e, inclusive, la atenuación puede ser mayor a la de estructuras vecinas. Si el QB está infectado o contiene secreciones espesas, la imagen puede semejar una tumoración sólida o, eventualmente, mostrar nivel hidroaéreo. ${ }^{20}$ Asimismo, la resonancia magnética (RM) (T1-T-2) aporta elementos importantes para el diagnóstico de presunción. Muchos autores consideran que con los elementos de imagen que proporcionan la combinación TC-RM, se puede considerar la presencia de QB con muchas posibilidades de certeza. ${ }^{7}$ EI US rutinario durante el embarazo puede detectar incidentalmente a la malformación, misma que aparece como una imagen quística anecoica en el tórax del feto. ${ }^{21} \mathrm{El}$ ecocardiograma ha descubierto incidentalmente QB en el corazón. ${ }^{22,23}$ Levine et al. ${ }^{24}$ publicaron un caso muy interesante de un incidentaloma descubierto por US en un feto de 15 semanas. La RM mostró una masa RM T2 de alta intensidad relacionada con la carina. En T1 no se hizo evidente, el quiste creció hasta obstruir ambos bronquios del feto, por lo que se decidió intervenir quirúrgicamente a las 33 semanas de gestación. El parto se llevó a cabo de tal manera que el producto siguió dependiendo de la placenta y del cordón umbilical mientras se afinaba el diagnóstico y se planeaba el tratamiento. La broncoscopia localizó la malformación sobre la carina en forma de $U$ invertida que se prolongaba hacia ambos bronquios, obstruyéndolos por completo. El QB se extirpó a través de la carina, que se reparó de inmediato (cirugía exútero-intraparto). Antes de seccionar el cordón umbilical, se instaló circulación extracorpórea empleando un oxigenador de membrana con objeto de dar tiempo a los pulmones del neonato a expandirse, terminar de madurar y producir el surfactante necesario. A los cuatro días se suspendió la circulación extracorpórea, y se pudo extubar a los siete. El neonato evolucionó bien. El caso constituye el primero reportado en la literatura de cirugía exúterointraparto. Existe un caso reportado de QB descubierto por tomografía con emisión de positrones (PET) en un paciente estudiado para detectar malignidad. ${ }^{25}$

Diagnóstico histológico. Nobuhara et al., ${ }^{26}$ en 1997, consideraron que el QB y la duplicación esofágica son producto de un mismo evento embriológico (la división del intestino primitivo), razón por la que ambas malformaciones pueden compartir características histológicas comunes, dando lugar en ocasiones a que la diferencia clínica sea difícil de establecer. Los autores revisaron 68 casos operados entre 1937 y 1995 en el Children's Hospital de la Universidad de Harvard.

La mayoría de los quistes presentaron epitelio respiratorio o glándulas bronquiales (58 de $68=75 \%$ ). En nueve niños se identificó epitelio gastroduodenal. Dos de ellos habían sido diagnosticados previamente como duplicación esofágica. Nobuhara al final mantuvo el diagnóstico de duplicación esofágica en 21 pacientes, basándose fundamentalmente en la localización intramural del quiste, aunque en 15 de los 21 casos (71\%) se identificó alguna evidencia de epitelio respiratorio, sustentando así, según los autores, el origen común de ambas malformaciones. Harvell et al. ${ }^{27}$ consideraron que los QB y los esofágicos pueden presentar epitelio ciliado, pero la presencia de dos capas de músculo liso con ausencia de glándulas respiratorias y de cartílago permite definir la presencia de quiste esofágico. Govaerts et al. ${ }^{28}$ señalaron que el diagnóstico histológico de QB se basa en la presencia de epitelio respiratorio típico, que aunado a la demostración de músculo liso, glándulas seromucosas o cartílago define el diagnóstico de QB. Más recientemente, Sharma et al. ${ }^{29}$ trataron de unificar la taxonomía y la clasificación de las formas quísticas derivadas del intestino primitivo unificando los conceptos embriopatológicos. Señalaron que los QB y los entéricos poseen similitudes en su origen y migración, pudiendo ambos ser atrapados en varios órganos. La diferencia entre los dos se debería a la etapa embriológica en que sucede el «pellizcamiento» en el brote ventral del intestino primitivo. En el caso del QB, tiene lugar durante la diferenciación bronquial, razón por la cual presenta elementos como glándulas y cartílago, mientras que los quistes entéricos «pellizcan» después de que la diferenciación bronquial ha terminado y, por lo tanto, no presentan ninguno de estos elementos. 
El quiste entérico y la duplicación esofágica tienen la misma apariencia histopatológica. La única diferencia sería la localización y la presencia o ausencia de la duplicación.

Tratamiento. El único tratamiento del QB es la extirpación quirúrgica. Los pacientes sintomáticos deben operarse de inmediato, en muchas ocasiones constituyen una verdadera emergencia. Los casos asintomáticos descubiertos incidentalmente y con sospecha de QB también deben operarse, tratando de prevenir complicaciones serias al hacerse sintomáticos, así como la eventual presencia de malignidad $(0.7-3 \%){ }^{30,31}$ No es posible predecir cuándo un portador asintomático se hará sintomático y qué tipo de cuadro presentará. Anteriormente sólo se vigilaba a este grupo de pacientes, hoy esto se ha desechado, ya que entre el 40 y $70 \%$ presentan manifestaciones clínicas en un plazo relativamente corto. EI QB debe extirparse por completo, pues se reportan recaídas cuando esto no se logra. ${ }^{32,33}$

Al presente, muchos grupos consideran a la videotoracoscopia como primera opción quirúrgica en casos bien seleccionados de adultos con QB mediastinal. Las ventajas que ofrece el procedimiento en cuanto a trauma, costo y recuperación son muy atractivas. El tamaño y adherencias de la tumoración a órganos vitales complican el procedimiento principalmente por sangrado, teniendo que recurrir a la toracotomía para completar el procedimiento. Martinod et al. ${ }^{34}$ reportaron 20 casos intentando de inicio el procedimiento endoscópico, teniendo que modificar la técnica a toracotomía en 7 casos por sangrado y adherencias. Weber et al. ${ }^{35}$ reportaron 20 casos en 7 años, teniendo que recurrir a cambio de abordaje en varios por dificultades técnicas y sangrado. Un grupo en la India ${ }^{36}$ comunicó 14 casos de QB de mediastino estudiados y operados durante 11 años. En 7 pacientes fue necesario abrir el tórax para completar el procedimiento. De Giacomo et al. ${ }^{37}$ en 13 años realizaron 30 procedimientos de resección de QB de mediastino en pacientes bien seleccionados, habiendo que recurrir únicamente en 2 casos a la toracotomía. Granato et al. ${ }^{38}$ trataron 30 casos de QB del mediastino entre 1975 y 2007 . En 25 casos emplearon toracotomía, encontrando casos muy complicados o con patología inusual. Únicamente operaron a 5 pacientes mediante toracoscopía; en uno tuvieron problemas de iatrogenia y en otro identificaron un carcinoma indiferenciado en la pared del quiste. Jiménez et al. ${ }^{39}$ reportaron 8 casos tratados con éxito mediante videotoracoscopia.

Algunos autores recomiendan puncionar el quiste cuando es posible, ya que así se facilita la disección endoscópica y la extracción de la tumoración. La mayoría de los casos pediátricos se siguen operando utilizando la toracotomía. En las localizaciones intraparenquimatosas se emplea la toracotomía con el procedimiento que sea necesario realizar en el pulmón. La punción del quiste se emplea como medida de descompresión temporal ante situaciones de emergencia.

\section{Prevalencia en casos operados. Información internacional}

Los QB son extraordinariamente raros, pero capaces de generar cuadros clínicos muy variados. Llama la atención que en los tratados de neumología o medicina interna prácticamente no se les menciona. En la literatura nacional encontramos únicamente siete comunicaciones que reúnen diez casos de QB, dos de ellos en localizaciones atípicas. Sorprende el ínfimo número de casos reportados, ya que en el país existen muchos centros hospitalarios que manejan volúmenes muy importantes de pacientes con patología cardiotorácica clínica y quirúrgica y, además, realizan gran cantidad de autopsias. La prevalencia real de esta patología se desconoce por los motivos ya mencionados.

Existen reportes en la literatura de instituciones reconocidas a nivel mundial, que revisan en forma retrospectiva el número de casos estudiados y tratados durante largos períodos de tiempo sin proporcionar las cifras del total de pacientes con otras patologías estudiados en el mismo lapso. Hacemos referencia a las citas nacionales, seguidas por las de las publicaciones foráneas que nos parecieron más interesantes. Medina et al..$^{40}$ publicaron un caso localizado en mediastino. Orozco et al. ${ }^{41}$ y Salcedo et al. ${ }^{42}$ dos casos cada uno de QB mediastinales. Domínguez et al. ${ }^{43}$ reportaron otros dos casos, uno en mediastino y el otro localizado en el lóbulo pulmonar inferior derecho. Quezada et al. ${ }^{44}$ diagnosticaron un caso prenatal mediante US, que se operó con éxito después de nacido. Guzmán et al. ${ }^{45}$ describieron un QB de localización atípica en la nasofaringe; por su parte, Anaya et al. ${ }^{46}$ reportaron otro caso atípico extraordinariamente infrecuente: se trató de un QB intramedular en un niño de 9 años que en tres meses había desarrollado cuadriplejia; se recuperó completamente después de extirpar la malformación. Kirmani et al. ${ }^{47}$ analizaron 683 casos de QB en adultos citados en 310 revistas médicas. Los pacientes sintomáticos se operaron de inmediato, así como los asintomáticos con imagenología sugestiva de QB, tratando de prevenir problemas severos durante el eventual inicio del cuadro: infección, ruptura, hemoptisis, etcétera, así como presencia de malignidad, que se encontró en cinco casos $(0.7 \%)$. Únicamente 74 individuos se vigilaron de manera conservadora durante un tiempo. De este grupo, se tuvo que intervenir a $33(45 \%)$ por inicio de 
sintomatología. La resección del quiste no aumentó la morbimortalidad. Suen et al. ${ }^{48}$ en un período de 30 años (1962-1991), encontraron en el Massachusetts General Hospital de Boston 42 casos: 37 mediastinales y cinco intraparenquimatosos; dos casos estuvieron asociados a malignidad. Sarper et al. ${ }^{49}$ analizaron 22 casos intervenidos quirúrgicamente entre 1985 y 2002 en el Texas Heart Institute, 18 hombres y 4 mujeres entre 1 y 38 años, con un promedio de edad de 16.4 años; encontraron 14 localizados en mediastino, dos hiliares y seis en pulmones. Ingresaron 18 pacientes con síntomas, 10 de ellos (45\%) con cuadros severos: hemoptisis, pleuritis, disfagia y neumotórax. Lee et al. ${ }^{50}$ en Corea, reportaron 24 pacientes estudiados entre 1991 y 2009 ; el $63 \%$ eran QB mediastinales, el resto $(37 \%)$ se encontraron localizados en pulmones. Fueron sintomáticos el $54.5 \%$ de los estudiados, y los asintomáticos fueron descubiertos incidentalmente por radiografías de tórax. McAdams et al. ${ }^{7}$ revisaron retrospectivamente 68 casos referidos por otras instituciones a los departamentos de patología-radiología del Instituto de las Fuerzas Armadas de Estados Unidos entre 1978 y 1997; predominaron los casos entre 0 y 29 años, siendo la edad promedio de 22; el paciente registrado con mayor edad fue de 72 años. El protocolo para definir a esta patología como QB requería que el estudio histológico de la pared del mismo mostrara los elementos que permiten determinar que la formación quística era un QB, a saber: epitelio respiratorio, glándulas secretoras, músculo liso y cartílago. Ribet et al., ${ }^{51}$ en Lille, Francia, revisaron 41 casos de QB localizados en pulmones de 20 adultos y 21 infantes-niños, cuatro de éstos diagnosticados en fase prenatal; se operaron 20 infantes-niños y 17 adultos; el $80 \%$ de los 41 casos eran asintomáticos; la mayoría desarrolló síntomas después de los 15 años. El mismo Ribet et al..$^{52}$ revisaron 69 casos de QB de mediastino estudiados en un lapso de 25 años con edades de 1-64 años. El 63.7\% presentaba síntomas que en el $43.4 \%$ se manifestaron como compresión a los órganos vecinos. Hubo dos casos con amenaza de muerte por la gravedad del cuadro clínico. Liu et al., ${ }^{53}$ en Beijing, reportaron 50 casos de QB entre los años 1983 y 2007; lograron la extirpación total de la malformación en 47 pacientes y parcial en tres. Cuypers et al..$^{54}$ publicaron los datos de 20 pacientes revisados entre 1975 y 1993, uno de ellos estaba asociado a carcinoma. Aktoğu et al..$^{55}$ reportaron 31 pacientes, 20 intraparenquimatosos y 11 mediastinales. En la revisión de Takeda et al. ${ }^{10}$ acerca de 105 casos de quistes del mediastino operados en el transcurso de 50 años, encontraron que esta patología representó el 13\% de las masas mediastinales, habiendo sido los QB los más frecuentes, con 47 casos. Shanti et al. ${ }^{2}$ revisaron
236 casos de quistes pulmonares tratados mediante resección pulmonar; en esta localización, también los QB fueron los más frecuentes: $20 \%$. St-Georges et al., ${ }^{56}$ en Canadá, reportaron 86 pacientes con edades entre 16 y 69 años portadores de QB, localizados 66 en el mediastino y 20 en el parénquima pulmonar y tratados en un lapso de 20 años. El diagnóstico de QB se sospechó en el $57 \%$ de los casos, pero en ninguno se logró hacer un diagnóstico de certeza en el preoperatorio; el $72 \%$ del total fueron sintomáticos. Todos los autores citados coincidieron en que la RM y la TC son los estudios que, combinándolos, tienen alrededor del 90-95\% de certeza diagnóstica en el QB, y que el único tratamiento de éstos es el quirúrgico que, además, está indicado de inmediato, tanto en los casos sintomáticos como en los asintomáticos, con objeto de prevenir cuadros clínicos complicados y, en algunas ocasiones, fatales.

Localizaciones atípicas. Se describen en la literatura aproximadamente 150 casos de QB en localizaciones atípicas, la mayoría de ellos relacionados con el desarrollo embriológico de la faringe y esófago. En cabeza y cuello se presentan generalmente como nódulos subcutáneos que pueden mimetizar a la perfección los cuadros clínicos que generarían los órganos con los que se relacionan, como los del tiroides. ${ }^{57}$ Mammadev et al. ${ }^{58}$ revisaron 70 citas de QB en cuello hallados en la literatura y describen el caso de un adolescente con una masa cervical que desplazaba el esófago. Tanita et al. ${ }^{59}$ describen la presencia de un QB subcutáneo en región escapular asociado a melanoma, y Ech-Cherif et al. ${ }^{60}$ publicaron un caso rarísimo localizado en la lengua. Las localizaciones atípicas en tórax están ligadas a órganos que durante el desarrollo embrionario se encuentran en la vecindad del árbol traqueobronquial. El timo, pericardio anterior y el primordio cardíaco descienden al tórax a partir de la octava semana de gestación, pudiendo en esta etapa incorporar en su estructura al QB ya formado. Tsai et al. ${ }^{61}$ informaron del caso de una mujer con un QB en el timo que evolucionó hacia la malignidad, y revisaron 22 casos de reportes en la literatura en niños y jóvenes con el mismo problema diagnóstico. El autor resalta que ningún procedimiento de imagenología es confiable para descartar la referida patología, por lo que recomienda intervenir quirúrgicamente ante cualquier sospecha de quiste en el timo, lo que apoyan también otros autores. ${ }^{62}$ Los QB que se localizan en el pericardio generalmente se manifiestan como un cuadro típico de pericarditis; ${ }^{63,64}$ o bien, por su tamaño son capaces de comprimir corazón y grandes vasos. La ruptura intrapericárdica de la malformación se manifiesta como hidropericardio, ${ }^{65}$ pudiendo incluso llegar a generar tamponade. Se anota la importancia del ecocardiograma transesofágico en estos casos. De Catte 
et al. ${ }^{22}$ trataron quirúrgicamente un QB localizado en el tabique interauricular del corazón; y Seo et al. ${ }^{23}$ describieron otro caso en la misma localización descubierto incidentalmente por imagenología. EI QB es capaz de originar arritmias cardiacas aun en ausencia de cardiopatía. Seo et al. ${ }^{66}$ describieron un caso de fibrilación auricular paroxística causada por un QB subcarinal que comprimía aurícula izquierda y venas pulmonares. La arritmia en estos casos se ha relacionado con las venas pulmonares. Parambil et al., ${ }^{67}$ mediante estudios de electrofisiología cardíaca, identificaron el foco ectópico en la desembocadura de la vena pulmonar inferior derecha, que se encontró elongada y distorsionada por un $\mathrm{QB}$ mediastinal posterior, precisamente en la desembocadura de esta vena a la aurícula izquierda. Las arritmias desaparecen al extirpar el quiste.

Encontramos en la literatura casos de QB hallados tanto en las paredes como en las cavidades ventriculares derecha e izquierda. Prates et al. ${ }^{68}$ describieron un caso en el ventrículo derecho. Weinrich et al. ${ }^{69}$ comunicaron el hallazgo incidental de un QB en el endocardio del ventrículo derecho durante el estudio ecocardiográfico en un cardiópata de 73 años. Wei et al., ${ }^{70}$ revisaron la literatura de QB localizados en corazón, encontraron 20 casos. El mismo autor relata el caso de un niño de cinco años con dolor precordial ocasionado por un QB epicárdico localizado en el ápex del ventrículo izquierdo que al resecarlo, se identificó el pedículo de la estructura, que llegaba hasta la cavidad ventricular. Señala que el dolor no era manifestación de isquemia, sino de mimetismo, pues al extirpar la malformación desapareció por completo el síntoma. Sumiyoshi et al. ${ }^{71}$ hipotéticamente hablaron acerca de la localización de los QB en el diafragma, abdomen y retroperitoneo, señalando que las cavidades abdominal y torácica están relacionadas al canal pericardio-peritoneal durante las primeras etapas embrionarias. Cuando el canal se divide en dos por la fusión de las membranas del canal pleuroperitoneal (el futuro diafragma), el intestino primitivo es «pellizcado» por esa estructura, explicando así la presencia de los QB en las mencionadas localizaciones. Esta teoría explicaría también la prevalencia de los QB en el retroperitoneo izquierdo, ya que el canal pericardio-peritoneal de este lado es más largo y se cierra después del derecho. En 1953 Miller reportó el primer caso de QB en el retroperitoneo; y recién Govaerts et al. ${ }^{28}$ revisaron la literatura mundial, encontrando 30 casos de QB retroperitoneales que reunían los criterios anatomopatológicos para considerarlos como tales. Reichelt et al., ${ }^{72}$ en 2000 , habían identificado únicamente 16 citas. Westphal et al. ${ }^{73}$ describieron un caso intradiafragmático. Jo et al..$^{74}$ encontraron un QB localizado en pleura posterior diafragmática izquierda que se extendía al retroperitoneo atravesando el diafragma, mientras Abdul et al. ${ }^{75}$ publicaron un caso de QB pedunculado en pleura. Ambos casos podrían apoyar el enfoque de Sumiyoshi et al..$^{71}$ Se anotan algunos de los casos de localización subdiafragmática que son más interesantes. En 1997, Doggett et al. ${ }^{76}$ describieron el caso de un QB en glándula suprarrenal izquierda que mimetizaba clínica y radiológicamente un feocromocitoma, no se ha encontrado explicación fehaciente hasta la fecha para este fenómeno. Yang et $a ._{.77}$ trataron de establecer bases desde el punto de vista de imagenología que pudieran orientar mejor hacia el diagnóstico de las masas retroperitoneales. En 2007, Yousset et al. ${ }^{78}$ describieron un QB localizado en páncreas y suprarrenal izquierda. Rubio et al. ${ }^{79}$ describieron un caso descubierto en la mucosa gástrica; y Sauvat et al. ${ }^{80}$ publicaron lo que el autor considera como el primer caso conocido en pediatría de un QB relacionado con el esófago abdominal, y que había sido diagnosticado como un tumor hepático. Orellana et al., ${ }^{81}$ en 2007, colectaron 39 casos de la literatura mundial de QB retroperitoneales y comunicaron otro caso con diámetro mayor de $10.8 \mathrm{~cm}$ localizado en suprarrenal izquierda. La gran variedad y lo bizarro de las manifestaciones sintomáticas, de acuerdo con la localización, tamaño y relación del QB con diferentes estructuras, así como su capacidad para mimetizar a la perfección múltiples cuadros clínicos, dan cabida a la intervención de varias especialidades médicas en el diagnóstico y tratamiento de la anomalía.

\section{CONCLUSIONES}

1. Los razonamientos que se plantean están basados únicamente en los datos que se obtuvieron de la revisión amplia de la literatura. Los QB son malformaciones congénitas extraordinariamente infrecuentes cuya prevalencia real se desconoce. Sin embargo, basándose en el número de casos reportados en las revisiones retrospectivas en relación con el lapso de años en que fueron estudiados, resultaría que se publican como promedio alrededor de $2.5-3$ casos de QB anuales.

2. Los casos sintomáticos se deben operar a la brevedad, algunos constituyen verdaderas urgencias. Al presente, existe unanimidad de criterio acerca de intervenir también quirúrgicamente los casos asintomáticos para prevenir la presencia de un cuadro agudo, grave y en ocasiones letal, así como la presencia de malignidad en el QB.

3. Los estudios que más orientan hacia el diagnóstico presuncional son los de imagenología, siendo los más importantes la TC y RM. El diagnóstico definitivo es el histológico de la pieza resecada. 
4. El único tratamiento definitivo en relación con los QB es la extirpación completa de la estructura. Los resultados resultan excelentes cuando se realiza a tiempo, pues aun los síntomas más complejos y espectaculares desaparecen.

5. Los cuadros clínicos que puede originar el QB pueden involucrar a médicos de muy distintas especialidades.

\section{REFERENCIAS}

1. Skandalakis JE, Gray SW, Symbas P. The trachea and the lung. In: Skandalakis JE, Gray SW, editors. Embryology for surgeons. 3rd ed. Baltimore, EUA: William \& Wilkins; 1994.p.414-450.

2. Shanti CM, Klein MD. Cystic lung disease. Semin Pediatr Surg 2008;17(1):2-8.

3. Tarpy SP, Kornfeld H, Moroz K, Lazar HL. Unusual presentation of a large tension bronchogenic cyst in an adult. Thorax 1993;48(9):951-952.

4. Basoglu A, Celik B, Sengul AT. Giant parenchymal bronchogenic cyst mimicking hydropneumothorax. J Thorac Cardiovasc Surg 2003;126(4):1201-1202.

5. Liu L, Pan T, Wei X. Bilateral giant pulmonary bronchogenic cysts. Asian Cardiovasc Thorac Ann 2009;17(1):6466. doi: 10.1177/0218492309102482.

6. Grewal RG, Yip CK. Intralobar pulmonary sequestration and mediastinal bronchogenic cyst. Thorax 1994;49(6):615-616.

7. McAdams HP, Kirejczyk WM, Rosado-de-Christenson $\mathrm{ML}$, Matsumoto S. Bronchogenic cyst: imaging features with clinical and histopathologic correlation. Radiology 2000;217(2):441-446.

8. Mee-Hye O, Eun Ah J, Ji Hye L, et al. Coexistence of intrapulmonary bronchogenic cyst and congenital cyst adenomatoid malformation. A case report. Korean J Pathol 2011;45(1):92-95.

9. MacKenzie TC, Guttenberg ME, Nisenbaum HL, Johnson MP, Adzick NS. A fetal lung lesion consisting of bronchogenic cyst, bronchopulmonary sequestration, and congenital cystic adenomatoid malformation: the missing link? Fetal Diagn Ther 2001;16(4):193-195.

10. Takeda S, Miyoshi S, Minami M, Ohta M, Masaoka A, Matsuda H. Clinical spectrum of mediastinal cysts. Chest 2003;124(1):125-132.

11. Kennebeck GA, Wong AK, Berry WR, Higgins JP, Manubens SM. Mediastinal bronchogenic cyst manifesting as a catastrophic myocardial infarction. Ann Thorac Surg 1999;67(6):1789-1791.

12. Eom DW, Kang GH, Kim JW, Ryu DS. Unusual bronchopulmonary foregut malformation associated with pericardial defect: bronchogenic cyst communicating with tubular esophageal duplication. J Korean Med Sci 2007;22(3):564-567.

13. Yoshida M, Kondo K, Toba H, Kenzaki K, Sakiyama S, Tangoku A. Two cases of bronchogenic cyst with severe adhesion to the trachea. J Med Invest 2007;54(1-2):187190.
14. Zaugg M, Kaplan V, Widmer U, Baumann PC, Russi EW. Fatal air embolism in an airplane passenger with a giant intrapulmonary bronchogenic cyst. Am J Respir Crit Care Med 1998;157(5 Pt 1):1686-1689.

15. Almeida FA, Desouza BX, Meyer T, Gregory S, Greenspon L. Intrapulmonary bronchogenic cyst and cerebral gas embolism in an aircraft flight passenger. Chest 2006;130(2):575-577.

16. Arnaiz J, Marco de Lucas E, Piedra T, Arnaiz Garcia ME, Patel AD, Gutierrez A. In-flight seizures and fatal air embolism: the importance of a chest radiograph. Arch Neurol 2011;68(5):661-664.

17. Edwardson M, Wurth D, Lacy JM, Fink J, Becker K. Cerebral air embolism resulting in fatal stroke in an airplane passenger with a pulmonary bronchogenic cyst. Neurocrit Care 2009;10(2):218-221.

18. Jung S, Wiest R, Frigerio S, Mattle HP, Hess CW. Cerebral air embolism caused by a bronchogenic cyst. Pract Neurol 2010;10(3):164-166. doi: 10.1136/jnnp.2010.211821.

19. Patel SR, Meeker DP, Biscotti CV, Kirby TJ, Rice TW. Presentation and management of bronchogenic cysts in the adult. Chest 1994;106(1):79-85.

20. Nakata H, Nakayama C, Kimoto T, et al. Computed tomography of mediastinal bronchogenic cysts. J Comput Assist Tomogr 1982;6(4):733-738.

21. Albright EB, Crane JP, Shackelford GD. Prenatal diagnosis of a bronchogenic cyst. J Ultrasound Med 1988;7(2):90-95.

22. De Catte L, De Backer T, Delhove O, Mares C. Ectopic bronchogenic cyst: sonographic findings and differential diagnosis. J Ultrasound Med 1995;14(4):321-323.

23. Seo N, Kang JW, Lim CH, Kim B, Lee HJ, Lim TH. CT findings of an intracardiac bronchogenic cyst. Int J Cardiovasc Imaging 2011;27(5):701-704.

24. Levine D, Jennings $R$, Barnewolt C, Mehta T, Wilson J, Wong G. Progressive fetal bronchial obstruction caused by a bronchogenic cyst diagnosed using prenatal MR imaging. AJR Am J Roentgenol 2001;176(1):49-52.

25. Sala E, Coulden R. Incidental bronchogenic cyst detected on F-18 FDG positron emission tomography. Clin Nucl Med 2004;29(8):494-495.

26. Nobuhara KK, Gorski YC, La Quaglia MP, Shamberger RC. Bronchogenic cysts and esophageal duplications: common origins and treatment. J Pediatr Surg 1997;32(10):1408-1413.

27. Harvell JD, Macho JR, Klein HZ. Isolated intra-abdominal esophageal cyst. A case report and review of the literature. Am Surg Pathol 1996;20(4):476-479.

28. Govaerts K, Van Eyken P, Verswijvel G, Van der Speeten $\mathrm{K}$. A bronchogenic cyst, presenting as a retroperitoneal cystic mass. Rare Tumors 2012;4(1): e13.

29. Sharma S, Nezakatgoo N, Sreenivasan P, Vanatta J, Jabbour N. Foregut cystic developmental malformation: new taxonomy and classification--unifying embryopathological concepts. Indian J Pathol Microbiology 2009;52(4):461-472.

30. Womack NA, Graham EA. Epithelial metaplasia in congenital cystic disease of the lung: Its possible relation to carcinoma of the bronchus. Am J Pathol 1941;17(5):645654.5 . 
31. Krous HF, Sexauer CL. Embryonal rhabdomyosarcoma arising within a congenital bronchogenic cyst in a child. J Pediatr Surg 1981;16(4):506-508.

32. Read CA, Moront M, Carangelo R, Holt RW, Richardson M. Recurrent bronchogenic cyst. An argument for complete surgical excision. Arch Surg 1991;126(10):1306-1308.

33. Hasegawa T, Murayama F, Endo S, Sohara Y. Recurrent bronchogenic cyst 15 years after incomplete excision. Interact Cardiovasc Thorac Surg 2003;2(4):685-687.

34. Martinod E, Pons F, Azorin J, et al. Thoracoscopic excision of mediastinal bronchogenic cysts: results in 20 cases. Ann Thorac Surg 2000;69(5):1525-1528.

35. Weber T, Roth TC, Beshay M, Herrmann P, Stein R, Schmid RA. Video-assisted thoracoscopic surgery of mediastinal bronchogenic cysts in adults: a single-center experience. Ann Thorac Surg 2004;78(3):987-991.

36. Panchanatheeswaran K, Dutta R, Singh KI, Kumar A. Eleven-year experience in thoracoscopic excision of bronchogenic cyst. Asian Cardiovasc Thorac Ann 2012;20(5):570-574.

37. De Giacomo T, Diso D, Anile M, et al. Thoracoscopic resection of mediastinal bronchogenic cysts in adults. Eur J Cardiothorac Surg 2009;36(2)357-359. doi: 10.1016/j. ejcts.2009.03.041

38. Granato F, Voltolini L, Ghiribelli C, Luzzi L, Tenconi S, Gotti G. Surgery for bronchogenic cysts: always easy? Asian Cardiovasc Thorac Ann 2009;17(5)467-471.

39. Jiménez Merchán R, Congregado Loscertales M, Gallardo Valera G, Ayarra Jarne J, Loscertales J. Resection of 8 mediastinal bronchogenic cysts by video-assisted thoracoscopy. Arch Bronconeumol 2008;44(4):220-223.

40. Medina MF, Echegoyen CR, Chavarría GJ, Morales FJ, Hernández GE, Barrera RR. Quiste broncogénico. Reporte de un caso clínico. Rev Inst Nal Enf Resp Méx 1995;8(3):236-239.

41. Orozco-Sánchez J, Sámano-Martínez A. Quiste broncogénico. Presentación de dos casos y revisión de la literatura. Bol Med Hosp Infant Mex 1992;49(7):449453.

42. Salcedo ChM, Alva LLF, Sotelo RR, Peña MES, Lule MMS, Falcón SV. Quiste broncogénico: reporte de dos casos y revisión de la literatura. Rev Inst Nal Enf Resp Mex 2004;17(1):35-41.

43. Domínguez-Flores R, Castillo-Ortega G. Comunicación de dos casos de quiste broncogénico y revisión de la literatura. Rev Inst Nal Enf Resp Méx 2005;18(3):217-220.

44. Quezada-Salazar CA, Navarrete-Arellano M. Quiste broncogénico, diagnóstico prenatal. Bol Med Hosp Infant Mex 2005;62(3):202-206.

45. Guzmán RE, García MO, Hernández CJ. Quiste broncogénico en la nasofaringe. Reporte de un caso y revisión bibliográfica. Rev Mex Pediatr 2000;67(5):227-230.

46. Anaya-Jara M, León-Ortiz A, Rueda-Franco F, MarhxBracho A. Quiste broncogénico intramedular. Acta Pediatr Mex 2010;31(4):158-161.

47. Kirmani B, Kirmani B, Sogliani F. Should asymptomatic bronchogenic cysts in adults be treated conservatively or with surgery? Interact Cardiovasc Thorac Surg 2010;11(5):649-659.
48. Suen HC, Mathisen DJ, Grillo HC, et al. Surgical management and radiologic characteristics of bronchogenic cysts. Ann Thorac Surg 1993;55(2):476-481.

49. Sarper A, Ayten A, Golbasi I, Demircan A, Isin E. Bronchogenic cyst. Tex Heart Inst J 2003;30(2);105-108.

50. Lee DH, Park CK, Kum DY, Kim JB, Hwang I. Clinical characteristics and management of intrathoracic bronchogenic cysts: a single center experience. Korean J Thorac Cardiovasc Surg 2011;44(4):279-284.

51. Ribet ME, Copin MC, Gosselin BH. Bronchogenic cysts of the lung. Ann Thorac Surg 1996;61(6):1636-1640.

52. Ribet ME, Copin MC, Gosselin BH. Bronchogenic cysts of the mediastinum. J Thorac Cardiovasc Surg 1995;109(5):1003-1010.

53. Liu HS, Li SH, Cao ZL, Zhang ZY, Ren H. Clinical features and treatment of bronchogenic cyst in adults. Chin Med Sci J 2009;24(1):60-63.

54. Cuypers P, De Leyn P, Cappelle L, Verougstraete L, Demedts M, Deneffe G. Bronchogenic cysts: a review of 20 cases. Eur J Cardiothorac Surg 1996;10(6):393396.

55. Aktoğu S, Yuncu G, Halilçolar H, Ermete S, Buduneli T. Bronchogenic cysts: clinicopathological presentation and treatment. Eur Respir J 1996;9(10):2017-2021.

56. St-Georges R, Deslauriers J, Duranceau A, et al. Clinical spectrum of bronchogenic cysts of the mediastinum and lung in the adult. Ann Thorac Surg 1991;52(1):613.

57. Ibáñez Aguirre J, Marti Cabane J, Bordas Rivas JM, Valenti Ponsa C, Erro Azcárate JM, De Simone P. A lump in the neck: cervical bronchogenic cyst mimicking a thyroid nodule. Minerva Chir 2006;61(1);71-72.

58. Mammadov E, Eliçevik M, Adaletli i, Dervişoğlu S, Celayir S. Bronchogenic cysts located in neck region: an uncommon entity with a common reason for misdiagnosis. Ann Pediatric Surg 2010;6(3)167-169.

59. Tanita M, Kikuchi-Numagami K, Ogoshi K, et al. Malignant melanoma arising from cutaneus bronchogenic cyst of the scapular area. J Am Acad Dermatol 2002;46(2 Suppl case reports):S19-S21.

60. Ech-Cherif El Kettani N, Dafiri R. Bronchogenic cyst of the tongue: a very rare localization. J Radiol 2004;(10 Pt 1):1736-1738.

61. Tsai JH, Lee JM, Lin MC, Liau JY. Carcinoid tumor arising in a thymic bronchogenic cyst associated with thymic follicular hyperplasia. Pathol Int 2012;62(1):49-54.

62. Donias HW, Chu QD, Anderson TM, Karamanoukian $\mathrm{RL}$, Gibbons WJ, Karamanoukian HL. Bronchogenic cysts presenting as thymic cysts. Contemp Surg 2002;58(11):562-565.

63. Kobza R, Oechslin E, Jenni R. An intrapericardial bronchogenic cyst. Interact Cardiovasc Thorac Surg 2003;2(3):279-280.

64. Davis M, Carneiro A, Dymarkowski S, Bogaert J. Bronchogenic cyst masquerading as pericarditis. Circulation 2010;122(7):e426-428.

65. Karangelis D, Tagarakis GI, Kalafati G, Tsilimingas NB. Spontaneous pneumopericardium as the first indication of an intrapericardial bronchogenic cyst. Tex Heart Inst J 2011;38(3):322. 
66. Seo N, Kang JW, Lim CH, Kim B, Lee HJ, Lim TH. CT findings of an intracardiac bronchogenic cyst. Int J Cardiovasc Imaging 2011;27(5):701-704.

67. Parambil JG, Gersh BJ, Knight MZ, Krowka MJ, Ryu JH. Bronchogenic cyst causing atrial fibrillation by impinging the right inferior pulmonary vein. Am J Med Sci 2006;331(6):336-368.

68. Prates PR, Lovato L, Homsi-Neto A, et al. Right ventricular bronchogenic cyst. Tex Heart Inst J 2003;30(1):71-73.

69. Weinrich M, Lausberg HF, Pahl S, Schäfers HJ. A bronchogenic cyst of the right ventricular endocardium. Am Thoracic Surg 2005;79(2):e13-14.

70. Wei X, Omo A, Pan T, Li J, Liu L, Hu M. Left ventricular bronchogenic cyst. Ann Thorac Surg 2006;81(4):e13-15.

71. Sumiyoshi K, Shimizu S, Enjoji M, Iwashita A, Kawakami K. Bronchogenic cyst in the abdomen. Virchows Arch A Pathol Anat Histopathol 1985;408(1):93-98.

72. Reichelt O, Grieser T, Wunderlich H, Möller A, Schubert J. Bronchogenic cyst. A rare differential diagnosis of retroperitoneal tumors. Urol Int 2000;64:216-219.

73. Westphal FL, Queiroz-Menezes A, Gonçalves GA. Intradiaphragmatic bronchogenic cyst. J Pneumologia 2003;29(3):148-150.

74. Jo WM, Shin JS, Lee IS. Supradiaphragmatic bronchogenic cyst extending into the retroperitoneum. Ann Thorac Surg 2006;81(1):369-370.

75. Abdul Rahman MR, Yaman MN, Dimon MZ, Zabir AF, Min JO, Hamid HA. Pedunculated parietal pleural lesion: a rare presentation of bronchogenic cyst. Ann Thorac Surg 2011;92(2):714-715.

76. Doggett RS, Carty SE, Clarke MR. Retroperitoneal bronchogenic cyst masquerading clinically and radiologically as a phaeochromocytoma. Virchows Arch 1997;431(1):73-76.

77. Yang DM, Jung DH, Kim H, et al. Retroperitoneal cystic masses: CT, clinical, and pathologic findings and literature review. Radiographics 2004;24(5):1353-1365.

78. El Youssef R, Fleseriu M, Sheppard BC. Adrenal and pancreatic presentation of subdiaphragmatic retroperitoneal bronchogenic cysts. Arch Surg 2010;145(3):302-304.

79. Rubio CA, Orrego A, Willén R. Congenital bronchogenic cyst in the gastric mucosa. J Clin Pathol 2005;58(3):335.

80. Sauvat F, Fusaro F, Jaubert F, Galifer B, Revillon Y. Paraesophageal bronchogenic cyst: first case reports in pediatric. Pediatr Surg Int 2006;22(10):849-851.

81. Orellana F, Cárdenas $R$, Manríquez ME, Ríos $H$, Suárez L, Videla D. Quiste broncogénico retroperitoneal: Caso clínico. Rev Méd Chile 2007;135(7):924-931.

\section{$\triangle$ Correspondencia:}

Dr. Miguel-Cosío Pascal, Hospital Ángeles Mocel.

Gelati No. 29-202. México, D.F. 11850.

Correo electrónico: micopa1311@aol.com

Los autores declaran no tener conflictos de interés. 